\title{
Lettre à la rédaction
}

\section{Efficacité de l'emploi tardif de la $\mathrm{N}$ acétyl cystéine dans l'intoxication au paracétamol}

\section{Efficacy of late administration of $\mathbf{N}$ acetyl cysteine in acetaminophen intoxication}

\author{
Catherine Coilliot ${ }^{1}$, Thierry Coton ${ }^{2 \star}$, Céline Ragot $^{3}$, Dominique Carre $^{2}$, Jean-Luc Moalic ${ }^{3}$, \\ Manuela Oliver ${ }^{3}$ \\ 1 Service de Pharmacie Hospitalière, Hôpital d'Instruction des Armées Laveran, BP 60149, 13384 Marseille Cedex 13, France \\ 2 Service de Gastro-entérologie, Hôpital d'Instruction des Armées Laveran, BP 60149, 13384 Marseille Cedex 13, France \\ ${ }^{3}$ Laboratoire de Biochimie, Hôpital d'Instruction des Armées Laveran, BP 60149, 13384 Marseille Cedex 13, France
}

\begin{abstract}
Mots clés : Acetaminophen, overdosage, antidote, $\mathrm{N}$ acetyl cysteine
Key words: Paracétamol, surdosage, antidote, $\mathrm{N}$ acétyl cystéine

Reçu le 25 septembre 2008, accepté après modifications le 5 février 2009

Publication en ligne le 26 mars 2009
\end{abstract}

Le paracétamol est un des médicaments les plus utilisés dans le monde, sa vente est libre dans la plupart des pays et la dose maximale recommandée est de $4 \mathrm{~g} / \mathrm{j}$. L'hépatite au paracétamol est préférentiellement rencontrée chez les adolescents et jeunes adultes sous forme isolée ou de poly-intoxication. La majorité des décès survient pour des populations plus âgées (plus de 40 ans) [1]. Son antidote, la N acétyl cystéine (NAC), utilisée de façon précoce (10 premières heures) a une efficacité remarquable.

Nous rapportons ici un cas d'intoxication aiguë grave au paracétamol chez un alcoolique chronique traité avec succès de cet antidote au-delà de la $10^{\mathrm{e}}$ heure.

Un homme de 42 ans était hospitalisé en urgence le 10 décembre 2003 dans le service de gastro-entérologie pour un tableau d'hépatite aiguë : il avait été admis la veille en service de psychiatrie pour l'aggravation récente de conduites d'alcoolisation.

Ses antécédents personnels étaient marqués par un éthylisme ancien et une toxicomanie à la buprénorphine (Subutex ${ }^{\circledR}$ ) sevrée depuis six mois. Son traitement habituel associait de la lévopromazine (Tercian $\left.{ }^{\circledR}\right)$, de l'alprazolam $\left(\right.$ Xanax ${ }^{\circledR}$ ), de la paroxétine (Divarius ${ }^{\circledR}$ ) et de l'alimémazine (Theralene ${ }^{\circledR}$ ).

À l'admission, le patient était apyrétique et son examen clinique était normal. Il n'y avait ni astérixis ni de trouble de la conscience. Son hémodynamique était stable et il n'existait

\footnotetext{
^ Correspondance : Dr Thierry Coton, thierrycoton@msn.com
}

aucun signe de choc. Le bilan biologique d'entrée révélait une macrocytose avec une thrombopénie, un cytolyse majeure avec des ALAT et des ASAT respectivement à 60 à 450 fois la normale, ainsi qu'une cholestase ictérique (phosphatases alcalines, gamma glutamyltransferase et bilirubine conjuguée respectivement à $1,5 \mathrm{~N}, 3 \mathrm{~N}, 75 \mu \mathrm{mol} / \mathrm{L})$. Le taux de prothrombine était effondré (23\%) avec un facteur $\mathrm{V}$ à $8 \%$. La fonction rénale était normale et une rhabdomyolyse était éliminée, les CPK étant normales. Les sérologies pour les virus des hépatites A, B, C, l'Epstein Barr virus, le cytomégalovirus et l'herpes simplex virus étaient négatives. L'échographie notait une hépato splénomégalie sans signe de dysmorphie hépatique ni de dilatation des veines sus hépatiques. La vésicule était alithiasique et il n'y avait pas de dilatation des voies biliaires.

L'ensemble de ces données permettait de retenir d'emblée le diagnostic d'hépatite aiguë sévère sans signe d'encéphalopathie hépatique. La présence d'une paracétamolémie à $29 \mathrm{mg} / \mathrm{L}$ mesurée plus de $24 \mathrm{~h}$ après l'admission en l'absence d'administration à l'hôpital, était le seul élément d'orientation étiologique et de gravité. Le patient avouait a posteriori la prise d'une quantité importante de Codoliprane ${ }^{\circledR}$ (paracétamol et codéine) les 8 et 9 décembre 2003, avec absorption respectivement de 6,4 et 12,8 g de paracétamol. Un traitement par la $\mathrm{N}$ acétyl cystéine était débuté le 10 décembre 2003 à la $24^{\mathrm{e}}$ heure d'hospitalisation $(150 \mathrm{mg} / \mathrm{kg}$ en une heure suivis de $150 \mathrm{mg} / \mathrm{kg}$ sur $20 \mathrm{~h}$ ) soit $48 \mathrm{~h}$ après le début de l'intoxication. L'évolution était favorable avec une normalisation du TP en 


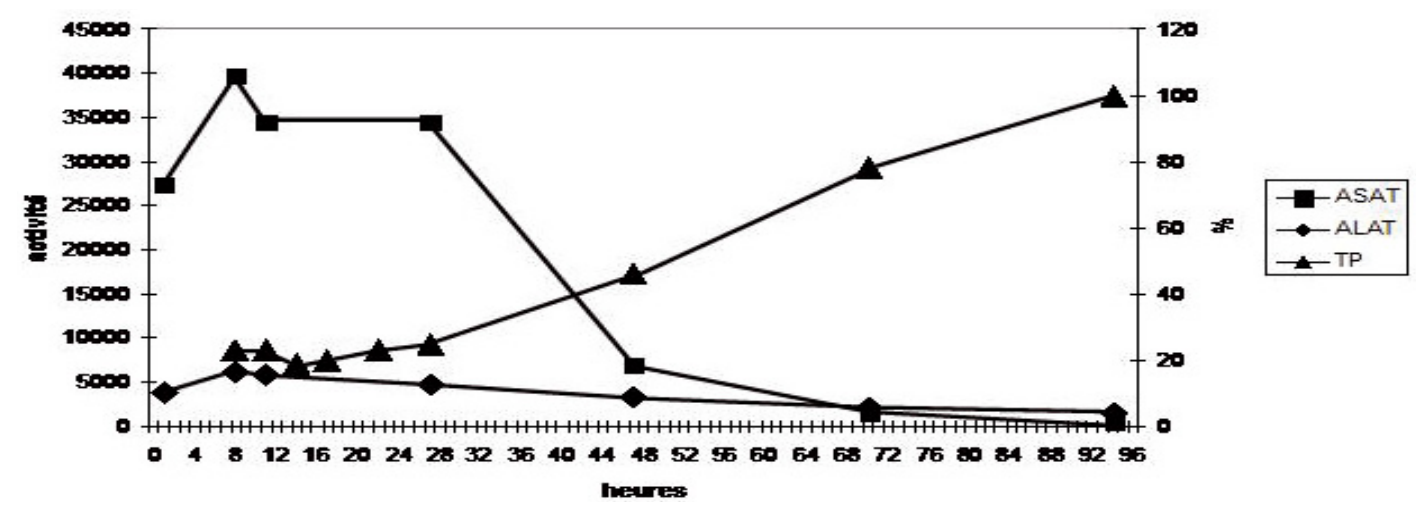

Fig. 1. Évolution des transaminases et du TP en fonction du temps.

$96 \mathrm{~h}$ et diminution significative des transaminases (figure 1) qui se normalisaient totalement en un mois. Le diagnostic d'hépatite aiguë sévère au paracétamol d'évolution favorable après administration tardive de NAC chez un éthylique chronique était finalement retenu.

La toxicité hépatique du paracétamol est bien connue en particulier chez le buveur chronique excessif [2]. Lors d'une intoxication massive, le glutathion est consommé. Lorsque $70 \%$ de la réserve en glutathion est épuisée, le $\mathrm{N}$ acétyl parabenzo quinone imine (NAPQI) s'accumule, conduisant à une nécrose des cellules hépatiques à prédominance centrolobulaire. La toxicité est dose dépendante. Classiquement, la dose toxique du paracétamol varie selon les auteurs entre 3 et $15 \mathrm{~g}$ chez l'adulte et 80 à $100 \mathrm{mg} / \mathrm{kg}$ chez l'enfant [3] et chez le buveur excessif des doses thérapeutiques $(3,5 \mathrm{~g})$ peuvent être hépatotoxiques [4]. Plus récemment, un consensus européen propose une dose toxique de $8 \mathrm{~g}$ chez l'adulte, de $200 \mathrm{mg} / \mathrm{kg}$ chez le nourrisson, de $150 \mathrm{mg} / \mathrm{kg}$ chez l'enfant de moins de 6 ans et de $100 \mathrm{mg} / \mathrm{kg}$ au-delà de 6 ans. La responsabilité du paracétamol dans notre cas d'hépatite a été retenue sur les données de l'interrogatoire, le profil de la cytolyse (ASAT > ALAT) et après élimination des causes usuelles d'hépatites aiguës (virus A, B, C, CMV, herpès) (la recherche d'hépatite $\mathrm{E}$ n'était pas systématique en 2003 en l'absence de séjour outremer), d'une insuffisance cardiaque droite, d'un état de choc et après réception des résultats de la paracétamolémie, la notion d'ingestion massive reconnue a posteriori par le patient certes polymédiqué (rôle aggravant possible de la codéine) et l'évolution rapidement favorable après administration de l'antidote. La recherche de toxique de type opiacés n'a pas été réalisée puisque l'interrogatoire seul retrouvait la notion de prise de codéine.

L'intoxication était biologiquement sévère chez notre patient qui présentait un TP à $23 \%$, un facteur $\mathrm{V}$ à $8 \%$ et une paracétamolémie de $29 \mathrm{mg} / \mathrm{L}$ plus de $24 \mathrm{~h}$ après la dernière prise. En effet, le TP et surtout le taux de facteur $\mathrm{V}$ ont une bonne valeur prédictive : un facteur $\mathrm{V}$ inférieur à $10 \%$ est associé à une évolution défavorable avec une sensibilité de $95 \%^{3}$. Enfin, la concentration toxique habituelle en paracétamol est de $200 \mathrm{mg} / \mathrm{L}$ à la quatrième heure et de $30 \mathrm{mg} / \mathrm{L}$ à la quinzième heure. La présence d'une acidose $(\mathrm{pH}<7.3)$, une créatininémie supérieure à $300 \mu \mathrm{mol} / \mathrm{L}$ sont aussi des facteurs péjoratifs [3]. L'âge, une atteinte hépatique chronique (virale), l'alcoolisme chronique, un long délai de prise en charge sont des facteurs aggravants indépendants. Ce dernier facteur est certainement le plus important car il conditionne la précocité de mise en œuvre du traitement antagoniste. Ces deux derniers facteurs péjoratifs étaient présents chez notre patient. Hay préconise que tout patient présentant une insuffisance hépatocellulaire aiguë doit recevoir précocement de la $\mathrm{N}$ acétyl cystéine (NAC) si une intoxication au paracétamol est suspectée, sans tenir compte du délai entre l'hospitalisation et l'ingestion [5], ce qui a été réalisé dans notre cas. Le délai médian est de $53 \mathrm{~h}$ (extrêmes : 36-80 h) dans la série de Keays [6]. En effet, la NAC permet de pallier le déficit en glutathion et donc d'assurer une détoxification correcte. Son utilisation, théoriquement fonction du délai écoulé depuis l'ingestion et de la cinétique de la paracétamolémie (normogramme de Rumack et Matthew) [7,8] est actuellement de plus en plus systématique en milieu hépatologique devant toute hépatite aiguë inexpliquée [4]. Son efficacité serait optimale dans les 8 premières heures en particulier en l'absence de cytolyse. Au delà de 24 h comme dans notre observation, la NAC ne permet plus de prévenir l'atteinte hépatique mais des études ont montré qu'elle permet de limiter la gravité de l'intoxication $[3,8,9]$.

Trois schémas de traitement sont proposés :

1) Celui de Prescott [10] qui a été utilisé dans notre observation, consiste en l'administration intraveineuse (IV) d'une dose de charge initiale de $150 \mathrm{mg} / \mathrm{kg}$ sur $15 \mathrm{~min}$ (60 min recommandées à l'heure actuelle) puis $50 \mathrm{mg} / \mathrm{kg}$ sur $4 \mathrm{~h}$ et enfin $100 \mathrm{mg} / \mathrm{kg}$ sur $16 \mathrm{~h}$, soit une dose totale de $300 \mathrm{mg} / \mathrm{kg}$ sur 21 h. Il est proposé actuellement de poursuivre ce protocole par une perfusion de $200 \mathrm{mg} / \mathrm{kg}$ sur $24 \mathrm{~h}$ en cas d'hypertransaminasémie même en l'absence de paracétamolémie détectable.

2) Celui de Smilkstein [11] administre une dose de charge initiale IV de $140 \mathrm{mg} / \mathrm{kg}$ puis $70 \mathrm{mg} / \mathrm{kg}$ toutes les $4 \mathrm{~h}$ à répéter 12 fois, soit une dose totale de $980 \mathrm{mg} / \mathrm{kg}$ sur $48 \mathrm{~h}$.

3) Enfin celui de Rumack [12] propose l'ingestion d'une dose initiale de charge de $140 \mathrm{mg} / \mathrm{kg}$ par voie orale puis $70 \mathrm{mg} / \mathrm{kg}$ toutes les $4 \mathrm{~h}$ à répéter 17 fois, soit une dose totale de $1330 \mathrm{mg} / \mathrm{kg}$ sur $68 \mathrm{~h}$. Des durées de traitement plus brèves (20 à $48 \mathrm{~h}$ ) ont été proposées récemment [13].

Si la voie orale est utilisée, l'emploi des ampoules injectables est plus adapté aux posologies recommandées. La voie orale n'est pas utilisable si le patient a reçu du charbon activé qui 
empêche l'absorption digestive de l'antidote ou si le patient présente des troubles de la conscience.

Ces trois schémas sont d'une efficacité équivalente si le traitement est instauré durant les 10 premières heures. Dans le cas où le traitement est instauré après la dixième heure, le schéma de Prescott fournit de moins bons résultats que les deux autres. En cas d'insuffisance hépatocellulaire, il convient de prolonger le traitement détoxifiant [14]. Dans l'étude de Louvet, où elle est employée systématiquement quelque soit le délai de prise en charge, la NAC est poursuivie jusqu'à l'obtention d'un TP supérieur à $50 \%$ [4]. Dans notre observation qui date de 2003, le protocole de Prescott a été appliqué à la lettre en dehors de la durée de l'administration de la dose initialement qui comme il est recommandé actuellement a été réalisée sur $60 \mathrm{~min}$.

Les études actuelles incitent à la prudence lors de l'utilisation du paracétamol à dose thérapeutique chez l'alcoolique et semblent indiquer une plus grande sévérité en cas de surdosage. Dans notre cas, l'intoxication est grave. En effet, la paracétamolémie mesurée au mieux à la $24^{\mathrm{e}}$ heure est de $29 \mathrm{mg} / \mathrm{L}$ et donc supérieure à $30 \mathrm{mg} / \mathrm{L}$ à la $15^{\mathrm{e}}$ heure si on se réfère au diagramme de Rumack et Matthew.

Des études expérimentales chez l'animal ont montré que l'administration aiguë de codéine entraîne une diminution des taux de glutathion dans les hépatocytes de rats et de souris $[15,16]$.

La déplétion en glutathion résultant du métabolisme de la codéine a aussi certainement contribué à aggraver, chez ce patient alcoolique, le pronostic de l'intoxication au paracétamol. Cette observation illustre :

1) l'intérêt d'un traitement même tardif (au-delà de la $10^{\mathrm{e}}$ heure) par la NAC, au cours d'une insuffisance hépatique aiguë dont l'origine ou une participation médicamenteuse doit toujours être évoquée [17];

2) la majoration de la gravité d'une intoxication au paracétamol chez les éthyliques chroniques ;

3) le rôle aggravant possible de la codéine dans la cytolyse et l'insuffisance hépatocellulaire.

\section{Références}

1. Schmidt LE. Age and paracetamol self-poisoning. Gut. 2005; 54: 686-690.

2. Schmidt LE, Dalhoff K, Poulsen HE. Acute versus chronic alcohol consumption in acetaminophen-induced hepatotoxicity. Hepatology. 2002; 35: 876-882.
3. Moritz F, Droy J. Le paracétamol. In: Danel V, Barriot P. Intoxications aiguës en réanimation. $2^{\mathrm{e}}$ édition. Rueil Malmaison : Arnette 1999 : 355-363.

4. Louvet A et al. L'alcool induit des mésaventures thérapeutiques au paracétamol et aggrave l'évolution en cas de surdosage : une étude prospective. Gastroenterol Clin Biol. 2008; 32: A25.

5. Hay JE. Acute Liver Failure. Curr Treat Options Gastroenterol. 2004; 7: 459-468.

6. Keays $\mathrm{R}$ et al. Intravenous acetylcysteine in paracetamol induced fulminant hepatic failure: a prospective controlled trial. BMJ. 1991; 303: 1026-1029.

7. Rumack BH, Matthew H. Acetaminophen poisoning and toxicity. Pediatrics. 1975; 55: 871-876.

8. Kozer E, Koren G. Management of paracetamol overdose: current controversies. Drug Saf. 2001; 24: 503-512.

9. Harrison PM, Keays R, Bray GP, Alexander GJ, Williams R. Improved outcome of paracetamol induce fulminant hepatic failure by late administration of acetylcysteine. Lancet. 1990; 336: 948.

10. Prescott LF, Illingworth RN, Critchley JA, Stewart MJ, Adam RD, Proudfoot FT. Intravenous N-acetylcysteine: the treatment of choice for paracetamol poisoning. BMJ. 1979; 2: $1097-$ 1100 .

11. Smilkstein MJ, Bronstein AC, Linden C, Augenstein WL, Kulig KW, Rumack BH. Acetaminophen overdose: a 48-hour intravenous $\mathrm{N}$-acetylcysteine treatment protocol. Ann Emerg Med. 1991; 20: 1058-1063.

12. Rumack BH, Peterson RG. Acetaminophen overdose: incidence, diagnosis and management in 416 patients. Pediatrics. 1978; 62: 898-903.

13. Betten DP, Cantrell FL, Thomas SC, Williams SR, Clarck RF. A prospective evaluation of shortened course oral $\mathrm{N}$ acetylcysteine for the treatment of acute acetaminophen poisoning. Ann Emerg Med. 2007; 50: 280-281.

14. Harrison PM, Wendon JA, Gimson AE, Alexander GJ, Williams R. Improvement by acetylcysteine of hemodynamics and oxygen transport in fulminant hepatic failure. N Engl J Med. 1991; 324: $1852-1857$.

15. Skoulis NP, James RC, Harbison RD, Roberts SM. Depression of hepatic glutathione by opioid analgesic drugs in mice. Toxicol Appl Pharmacol. 1989; 99: 139-147.

16. Ellington SP, Rosen GM. Codeine-mediated hepatotoxicity in isolated rat hepatocytes. Toxicol Appl Pharmacol. 1987; 90: $156-165$.

17. Wolf SJ, Heard K, Sloan EP, Jagoda AS. American college of emergency physicians. Clinical policy: critical issues in the management of patients presenting to the emergency department with acetaminophen overdose. Ann Emerg Med. 2007; 50: 292-313. 